\title{
Deeply virtual Compton scattering on a virtual pion target
}

\author{
D. Amrath ${ }^{1}$, M. Diehl ${ }^{1, a}$, J.P. Lansberg ${ }^{2,3}$ \\ ${ }^{1}$ Deutsches Elektronen-Synchroton DESY, 22603 Hamburg, Germany \\ ${ }^{2}$ Centre de Physique Théorique, École Polytechnique, CNRS, 91128 Palaiseau, France \\ ${ }^{3}$ Institut für Theoretische Physik, Universität Heidelberg, Philosophenweg 19, 69120 Heidelberg, Germany
}

Received: 8 August 2008 / Published online: 14 October 2008

(C) Springer-Verlag / Società Italiana di Fisica 2008

\begin{abstract}
We study deeply virtual Compton scattering on a virtual pion that is emitted by a proton. Using a range of models for the generalized parton distributions of the pion, we evaluate the cross section, as well as the beam spin and beam charge asymmetries in the leading-twist approximation. Studying Compton scattering on the pion in suitable kinematics puts high demands on both beam energy and luminosity, and we find that the corresponding requirements will first be met after the energy upgrade at Jefferson Laboratory. As a by-product of our study, we construct a parameterization of pion generalized parton distributions that has a non-trivial interplay between the $x$ and $t$ dependence and is in good agreement with form factor data and lattice calculations.
\end{abstract}

PACS 12.38.-t $\cdot$ 13.60.-r $\cdot$ 14.40.Aq

\section{Introduction}

The concept of generalized parton distributions (GPDs) is a versatile tool to describe hadron structure at the quark-gluon level and has given rise to vigorous theoretical and experimental activities. Among the attractive features of GPDs are the possibilities to connect ordinary parton densities with elastic form factors [1] and to explore the spatial distributions of partons inside a hadron [2-5]. Reviews of this extensive subject can be found in [6-9].

The pion plays a special role in the low-energy sector of QCD as the lightest bound state and the pseudo-Goldstone boson associated with chiral symmetry breaking. Given the difficulty to perform high-energy experiments with a pion in the initial state, our knowledge of its internal structure is, however, scarce compared with what is known about the nucleon. Currently, the principal sources of information are the

a e-mail: markus.diehl@desy.de spacelike electromagnetic form factor of the pion [10-15] and its parton densities extracted from Drell-Yan production with pion beams [16-18]. Measurements constraining the GPDs of the pion would provide a natural extension of this knowledge. On the theoretical side, the pion GPDs have been studied in a number of dynamical models [19-29] and on the lattice [30-33]. Important theoretical investigations have been performed for a pion target in the first instance, because of its relative simplicity as a spin-zero particle, see for instance [34-39].

The purpose of the present work is to estimate how pion GPDs may be investigated in deeply virtual Compton scattering (DVCS), which is the theoretically cleanest and most advanced among the hard exclusive processes involving generalized parton distributions. We consider the reaction $e p \rightarrow e \gamma \pi^{+} n$ at small invariant momentum transfer between the proton and neutron. In the one-pion exchange approximation, the reaction is then described by the emission of a slightly off-shell pion from the proton, followed by the scattering process $e \pi^{+} \rightarrow e \gamma \pi^{+}$. This can be seen as an analog of the reaction $e p \rightarrow e \pi^{+} n$, which has been used to extract the electromagnetic pion form factor for all but the smallest values of momentum transfer [11-15]. Two mechanisms contribute to $e \pi \rightarrow e \gamma \pi$, namely virtual Compton scattering and the Bethe-Heitler process, as shown in Fig. 1. Suitable strategies for isolating the Compton signal are the same as those for scattering on a nucleon target, which have been elaborated in detail $[40,41]$ and successfully used in experiment [42-48]. The corresponding expressions for the pion can be found in [49], where also numerical estimates for $e \pi \rightarrow e \gamma \pi$ have been given. We note that in suitable kinematics, the reaction $e p \rightarrow e \gamma \pi^{+} n$ may also be used to study virtual Compton scattering on the pion in the backward region, whose description involves the so-called transition distribution amplitude from a pion to a photon [5052]. In experiments with a real photon beam, the lepton-pair production process $\gamma p \rightarrow e^{+} e^{-} \pi^{+} n$ can provide access to 

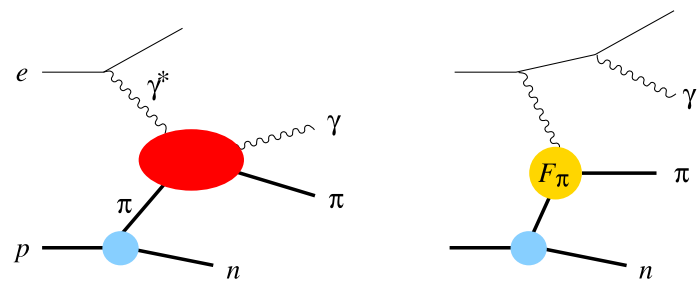

Fig. 1 Graphs for $e p \rightarrow e \gamma \pi^{+} n$ in the one-pion exchange approximation. Contributing subprocesses are virtual Compton scattering on a pion (left) and the Bethe-Heitler process (right). The crossed Bethe-Heitler graph (not shown) has the photons attached to the lepton line in opposite order. The blob marked with $F_{\pi}$ represents the electromagnetic pion form factor

timelike Compton scattering $\gamma \pi \rightarrow \gamma^{*} \pi$ on the pion, which is closely related to DVCS by crossing [53].

Our paper is organized as follows. In the next section we present in some detail the kinematics of $e p \rightarrow e \gamma \pi^{+} n$. In Sect. 3 we give the basic equations for the analysis of this process in the one-pion exchange picture and in the framework of generalized parton distributions. We also briefly discuss the validity of the one-pion exchange approximation. In Sect. 4 we present a model for the GPDs of the pion, paying special attention to their $t$-dependence. In Sect. 5 we finally give estimates for cross sections and asymmetries in the HERMES experiment and at Jefferson Lab, before summarizing our main findings in Sect. 6.

\section{Kinematics}

In this section we discuss the kinematics of the reaction

$e(l)+p(p) \rightarrow e\left(l^{\prime}\right)+\gamma\left(q^{\prime}\right)+\pi^{+}\left(p_{\pi}^{\prime}\right)+n\left(p^{\prime}\right)$,

with four-momenta given in parentheses. We write

$q=l-l^{\prime}, \quad p_{\pi}=p-p^{\prime}$

for the four-momenta of the virtual photon and the virtual pion, and use the standard variables

$$
\begin{array}{ll}
Q^{2}=-q^{2}, & W^{2}=(p+q)^{2}, \quad s=(p+l)^{2}, \\
x_{B}=\frac{Q^{2}}{2 p \cdot q}, & y=\frac{p \cdot q}{p \cdot l}
\end{array}
$$

for deep inelastic scattering processes. The variables

$t=\left(p-p^{\prime}\right)^{2}, \quad x_{\pi}=\frac{p_{\pi} \cdot l}{p \cdot l}$

describe the emission of the virtual pion from the proton target, where $x_{\pi}$ is the fraction of energy that the virtual pion takes away from the proton in the $e p$ c.m. The azimuthal angles of the final-state electron and neutron in that frame are respectively denoted by $\psi_{e}$ and $\psi_{n}$, where the $z$-axis is chosen along the lepton beam momentum. We write $m_{N}$ and $m_{\pi}$ for the nucleon and pion masses, and neglect the lepton mass throughout our work. An important role is played by the kinematic limit

$-t \geq-t_{0}=\frac{x_{\pi}^{2} m_{N}^{2}}{1-x_{\pi}}$

which is readily obtained from the expression $\boldsymbol{p}_{T}^{\prime 2}=-t(1-$ $\left.x_{\pi}\right)-x_{\pi}^{2} m_{N}^{2}$ for the squared transverse momentum of the neutron in the ep c.m. We need two more variables to describe the $\gamma$ and $\pi$ in the final state, namely

$t_{\pi}=\left(p_{\pi}-p_{\pi}^{\prime}\right)^{2}$

and the azimuthal angle $\phi_{\pi}$ between the plane spanned by $\boldsymbol{p}_{\pi}$ and $\boldsymbol{p}_{\pi}^{\prime}$ and the plane spanned by $\boldsymbol{l}$ and $\boldsymbol{l}^{\prime}$ in the c.m. of $\pi \gamma$ in the final state. For the sign of $\phi_{\pi}$ we follow the usual convention for DVCS on a proton target. ${ }^{1}$ For our later discussion it is useful to introduce further variables, which refer to the subprocess $e \pi \rightarrow e \gamma \pi$ on the virtual pion target, namely

$s_{\pi}=\left(p_{\pi}+q\right)^{2}, \quad x_{B}^{\pi}=\frac{Q^{2}}{2 p_{\pi} \cdot q}, \quad y_{\pi}=\frac{p_{\pi} \cdot q}{p_{\pi} \cdot l}$.

One finds

$$
\begin{aligned}
s_{\pi}= & Q^{2}\left(\frac{x_{\pi}}{x_{B}}-1\right)+2 y x_{B} x_{\pi} m_{N}^{2}+\left(1-y x_{B}\right) t \\
- & 2 \cos \left(\psi_{e}-\psi_{n}\right) \sqrt{(1-y) Q^{2}-\left(y x_{B} m_{N}\right)^{2}} \\
& \times \sqrt{\left(1-x_{\pi}\right)\left(t_{0}-t\right)} .
\end{aligned}
$$

To select kinematics where DVCS on a pion can be described in terms of generalized parton distributions, we take the Bjorken limit

$Q^{2} \rightarrow \infty$ at fixed $y, x_{B}, x_{\pi}, t, t_{\pi}$.

The squared c.m. energies $s, W^{2}$, and $s_{\pi}$ then become large, whereas the squared momentum transfers $t$ and $t_{\pi}$ are small compared with $Q^{2}$. The relation (8) then implies

$x_{B}^{\pi} \approx \frac{x_{B}}{x_{\pi}}, \quad y_{\pi}=y \frac{x_{B}}{x_{\pi} x_{B}^{\pi}} \approx y$

and

$x_{\pi} y \approx \frac{s_{\pi}+Q^{2}}{s}$

\footnotetext{
${ }^{1}$ One thus obtains $\phi_{\pi}$ from the angle $\phi_{h}$ in [54] by replacing $P \rightarrow p_{\pi}$ and $P_{h} \rightarrow p_{\pi}^{\prime}$.
} 
with corrections of order $m / Q$, where $m^{2}$ represents the small scales $m_{N}^{2}, m_{\pi}^{2}, t$ and $t_{\pi}$, whose magnitudes we do not distinguish at this point. The phase space element of the process (1) can be written as

$$
\begin{gathered}
\frac{d^{3} l^{\prime}}{2 l^{\prime 0}} \frac{d^{3} q^{\prime}}{2 q^{\prime 0}} \frac{d^{3} p_{\pi}^{\prime}}{2 p_{\pi}^{\prime 0}} \frac{d^{3} p^{\prime}}{2 p^{\prime 0}} \delta^{(4)}\left(l^{\prime}+q^{\prime}+p_{\pi}^{\prime}+p^{\prime}-l-p\right) \\
=\frac{d Q^{2} d y d \psi_{e} d t_{\pi} d \phi_{\pi} d t d x_{\pi} d \psi_{n}}{64 \sqrt{\left(s_{\pi}+Q^{2}+t\right)^{2}-4 s_{\pi} t}} .
\end{gathered}
$$

The interpretation of the process (1) in terms of DVCS on a virtual pion target puts several conditions on the kinematics, which we now discuss. First of all we impose an upper cutoff on $|t|$,

$|t| \leq|t|_{\max }$

to ensure that the $p \rightarrow n$ transition is dominated by virtual pion emission. Since $|t|_{\max }$ must be bigger than $-t_{0}$ in (5), this implies a maximum value for $x_{\pi}$,

$x_{\pi} \leq x_{\pi \max }=\frac{1}{2}\left[\sqrt{\tau^{2}+4 \tau}-\tau\right]$

with $\tau=|t|_{\max } / m_{N}^{2}$. Since we want the subprocess $\gamma^{*} \pi \rightarrow$ $\gamma \pi$ to be in Bjorken kinematics, we further impose lower cutoffs

$s_{\pi} \geq s_{\pi \min }, \quad Q^{2} \geq Q_{\min }^{2}$.

According to (11) this implies lower limits on $x_{\pi}$ and on $y$,

$$
\begin{aligned}
& x_{\pi \min } \approx \frac{1}{y_{\max }} \frac{s_{\pi \min }+Q_{\min }^{2}}{s}, \\
& y_{\min } \approx \frac{1}{x_{\pi \max }} \frac{s_{\pi \min }+Q_{\min }^{2}}{s},
\end{aligned}
$$

where $y_{\max }$ is an upper limit on $y$ we will later impose both for theoretical and for experimental reasons (see Sect. 5). The relation (11) also restricts the largest possible values of $Q^{2}$ to

$Q_{\max }^{2} \approx s x_{\pi \max } y_{\max }-s_{\pi \min }$.

For a clean physical interpretation of the reaction (1) as DVCS on a weakly off-shell pion target, it is desirable to take rather small $|t|_{\max }$ and rather large $s_{\pi \min }$ and $Q_{\min }^{2}$. With (14) and the first relation in (16), this only leaves enough phase space for $x_{\pi}$ if the total c.m. energy $s$ is sufficiently large.

\section{Calculation of the cross section}

In this section we give some basic formulae for the process $e p \rightarrow e \gamma \pi n$ in the one-pion exchange approximation and discuss the validity of this approximation.
3.1 The one-pion exchange approximation

In the one-pion exchange approximation, the amplitudes for $e p \rightarrow e \gamma \pi n$ and for $e \pi \rightarrow e \gamma \pi$ are related as

$$
\begin{aligned}
& \mathcal{M}_{e p}=\bar{u}\left(p^{\prime}\right) \gamma_{5} u(p) \frac{\sqrt{2} g_{\pi N N}}{m_{\pi}^{2}-t} F(t) \mathcal{M}_{e \pi}, \\
& F(t)=\frac{\Lambda^{2}-m_{\pi}^{2}}{\Lambda^{2}-t}
\end{aligned}
$$

with the pion-nucleon coupling $g_{\pi N N}=13.05$ [55]. Here we have introduced a phenomenological factor $F(t)$ to soften the pion-nucleon vertex when the pion virtuality $t$ becomes large compared to $m_{\pi}^{2}$. In our calculations we will take $\Lambda=800 \mathrm{MeV}$ from [56]. The $e p$ cross section is then given by

$$
\begin{aligned}
& \frac{d^{8} \sigma(e p \rightarrow e \gamma \pi n)}{d y d Q^{2} d \psi_{e} d t_{\pi} d \phi_{\pi} d t d x_{\pi} d \psi_{n}} \\
& =\frac{1}{128(2 \pi)^{8}\left(s-m_{N}^{2}\right) \sqrt{\left(s_{\pi}+Q^{2}+t\right)^{2}-4 s_{\pi} t}} \\
& \quad \times\left[\sqrt{2} g_{\pi N N} F(t)\right]^{2} \frac{-t}{\left(m_{\pi}^{2}-t\right)^{2}} \sum_{\text {spins }}\left|\mathcal{M}_{e \pi}\right|^{2}
\end{aligned}
$$

where $\sum_{\text {spins }}$ sums over the polarizations of the final-state electron and photon. In (19) we have further averaged over the polarization of the proton target and summed over the polarization of the outgoing neutron, but kept the lepton beam polarization fixed. Defining the cross section

$$
\begin{aligned}
& \frac{d^{4} \sigma(e \pi \rightarrow e \gamma \pi)}{d y_{\pi} d Q^{2} d t_{\pi} d \phi_{\pi}} \\
& =\frac{1}{32(2 \pi)^{4} x_{\pi}\left(s-m_{N}^{2}\right)} \\
& \quad \times \frac{1}{\sqrt{\left(s_{\pi}+Q^{2}+t\right)^{2}-4 s_{\pi} t}} \sum_{\text {spins }}\left|\mathcal{M}_{e \pi}\right|^{2}
\end{aligned}
$$

on a virtual pion target, we have the simple relation

$$
\begin{aligned}
& \frac{d^{6} \sigma(e p \rightarrow e \gamma \pi n)}{d y d Q^{2} d t_{\pi} d \phi_{\pi} d t d x_{\pi}} \\
& =x_{\pi} \frac{g_{\pi N N}^{2}}{8 \pi^{2}}[F(t)]^{2} \frac{-t}{\left(m_{\pi}^{2}-t\right)^{2}} \\
& \times \frac{d^{4} \sigma(e \pi \rightarrow e \gamma \pi)}{d y_{\pi} d Q^{2} d t_{\pi} d \phi_{\pi}},
\end{aligned}
$$

where we have integrated over the angles $\psi_{e}$ and $\psi_{n}$.

For a rough estimate one may neglect the dependence of the $e \pi$ cross section (20) on the pion virtuality $t$. Integrating 


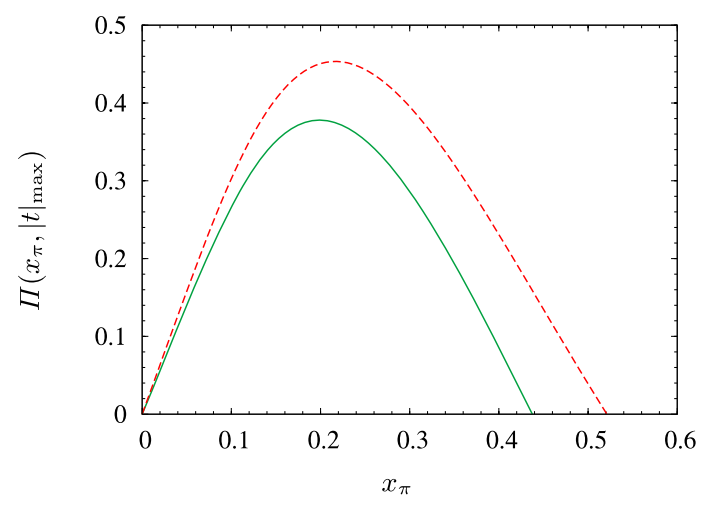

Fig. 2 The pion flux factor $\Pi\left(x_{\pi},|t|_{\max }\right)$ defined in (23), shown for $|t|_{\max }=0.3 \mathrm{GeV}^{2}$ (solid) and $|t|_{\max }=0.5 \mathrm{GeV}^{2}$ (dashed)

the factor of proportionality in (21) over $t$, one then obtains

$$
\begin{aligned}
& \frac{d^{4} \sigma(e p \rightarrow e \gamma \pi n)}{d y d Q^{2} d t_{\pi} d \phi_{\pi}} \\
& \quad \approx \int d x_{\pi} \Pi\left(x_{\pi},|t|_{\max }\right) \frac{d^{4} \sigma(e \pi \rightarrow e \gamma \pi)}{d y_{\pi} d Q^{2} d t_{\pi} d \phi_{\pi}}
\end{aligned}
$$

with

$$
\Pi\left(x_{\pi},|t|_{\max }\right)=x_{\pi} \frac{g_{\pi N N}^{2}}{8 \pi^{2}} \int_{-|t|_{\max }}^{t_{0}\left(x_{\pi}\right)} d t[F(t)]^{2} \frac{-t}{\left(m_{\pi}^{2}-t\right)^{2}},
$$

where $t_{0}\left(x_{\pi}\right)$ is given in (5). Note that the $e \pi$ cross section on the r.h.s. of (22) depends on $x_{\pi}$ via $s_{\pi} \approx x_{\pi} y_{\pi} s-Q^{2}$. With the form factor of the pion-nucleon vertex taken in (18), the integral in (23) can be done analytically using

$$
\begin{aligned}
& \int d t[F(t)]^{2} \frac{-t}{\left(m_{\pi}^{2}-t\right)^{2}} \\
& =\frac{\Lambda^{2}+m_{\pi}^{2}}{\Lambda^{2}-m_{\pi}^{2}} \ln \frac{\Lambda^{2}-t}{m_{\pi}^{2}-t}-\frac{\Lambda^{2}}{\Lambda^{2}-t}-\frac{m_{\pi}^{2}}{m_{\pi}^{2}-t} .
\end{aligned}
$$

In Fig. 2 we show the pion flux factor $\Pi\left(x_{\pi},|t|_{\max }\right)$ as a function of $x_{\pi}$ for two values of $|t|_{\max }$.

\subsection{Validity of the one-pion exchange approximation}

The validity of the one-pion exchange approximation in the process (1) cannot be taken for granted, and it is natural to see what is known for similar processes.

As already mentioned in the introduction, an important process in this context is $e p \rightarrow e \pi n$, where the one-pion exchange approximation yields the subprocess $e \pi \rightarrow e \pi$, from which the electromagnetic pion form factor can be extracted. The most recent measurements [14, 15], as well as previous data, provide a clear indication that pion exchange cannot be the only contribution to this reaction. This is because the cross section $\sigma_{T}$ for $\gamma^{*} p \rightarrow \pi n$ with transverse $\gamma^{*}$ polarization in the $\gamma^{*} p$ c.m. is seen to be clearly nonzero, although at low $|t|$ it is smaller than the cross section $\sigma_{L}$ for a longitudinal $\gamma^{*}$. To understand the implications of this observation, let us take the case $|t|=|t|_{\text {min }}$, where by definition the angle between the proton and neutron momenta is zero in the $\gamma^{*} p$ c.m. Due to angular momentum conservation, the subprocess $\gamma^{*} \pi \rightarrow \pi$ can then only proceed for longitudinal photon polarization, i.e., pion exchange contributes only to $\sigma_{L}$. In terms of $t$-channel exchanges, the presence of $\sigma_{T}$ is thus an indicator for the exchange of states with nonzero spin, with the $\rho$ being an obvious candidate because of its relatively low mass. A corresponding model calculation in [57] indeed yields a nonvanishing $\sigma_{T}$, although it undershoots the measured values of [14] and [15], which were taken at $\gamma^{*} p$ c.m. energies of $W=1.95 \mathrm{GeV}$ and $W=2.22 \mathrm{GeV}$, respectively. In [14] this mismatch was ascribed to possible resonance contributions in the $\pi n$ channel. At $|t|=|t|_{\min }$ there is no contribution to $\sigma_{L}$ from $\rho$ exchange because of parity conservation in the subprocess $\gamma^{*} \rho \rightarrow \pi$, so that for the extraction of the pion form factor a separation of $\sigma_{L}$ and $\sigma_{T}$ should considerably enhance the contribution from pure pion exchange.

We note that the situation in $\gamma^{*} p \rightarrow \gamma \pi n$ is different. Even at $|t|=|t|_{\min }$ both subprocesses $\gamma^{*} \pi \rightarrow \gamma \pi$ and $\gamma^{*} \rho \rightarrow \gamma \pi$ can take place for transverse $\gamma^{*}$ polarization, which is dominant in Bjorken kinematics according to the factorization theorem for DVCS. Whereas taking low $|t|$ will enhance pion exchange also in this case, it may be useful to assess the quantitative importance of $\gamma^{*} \rho \rightarrow \gamma \pi$. This would require information about the GPDs for the $\rho \rightarrow \pi$ transition. Simple helicity counting [58] shows that at twisttwo level there are two GPDs involving the axial current and one GPD involving the vector current for the quarks. Their $x$ moments are accessible to an evaluation in lattice QCD, which may thus help to estimate the role of $\rho$ exchange in the reaction $e p \rightarrow e \gamma \pi n$. In particular, the lowest moment of the vector current GPD gives the electromagnetic $\rho \rightarrow \pi$ transition form factor, which also enters the Bethe-Heitler process in $e \rho \rightarrow e \gamma \pi$. A further constraint on the $\rho \rightarrow \pi$ transition GPDs can be obtained by taking the limit of soft pion momentum, in analogy to what has been done for the $N \rightarrow \pi$ transition in [59].

Concerning resonance contributions, we recall that the $\gamma^{*} p$ c.m. energy $W$ is large in Bjorken kinematics. In experiments suited to investigate DVCS, $W$ will thus be much larger than in the pion form factor measurements [14, 15]. More problematic are possible resonances in the $\pi n$ channel of the final state, which are of course not taken into account by the one-pion exchange approximation. The relevant invariant mass is 


$$
\begin{aligned}
M_{\pi n}^{2}= & \left(p_{\pi}^{\prime}+p^{\prime}\right)^{2}=m_{N}^{2}+2 m_{N} E_{\pi}+t_{\pi}-t \\
= & \frac{1}{x_{\pi}}\left[\left(t_{\pi 0}-t_{\pi}\right)\left(1-x_{\pi}\right)-t\left(1-x_{B}^{\pi}\right)+\frac{1-x_{\pi}}{1-x_{B}^{\pi}} m_{\pi}^{2}\right. \\
& +2 \cos \left(\phi_{\pi}+\psi_{e}-\psi_{n}\right) \sqrt{\left(1-x_{\pi}\right)\left(1-x_{B}^{\pi}\right)} \\
& \left.\times \sqrt{\left(t_{0}-t\right)\left(t_{\pi 0}-t_{\pi}\right)}\right] \\
& +m_{N}^{2}+m_{\pi}^{2}+\mathcal{O}\left(\frac{m^{3}}{Q}\right) .
\end{aligned}
$$

Here $E_{\pi}$ is the energy of the outgoing pion in the proton rest frame, and $t_{\pi 0}$ is the upper kinematic limit of $t_{\pi}$. In the Bjorken limit one has

$t_{\pi 0} \approx x_{B}^{\pi} t-\frac{x_{B}^{\pi} m_{\pi}^{2}}{1-x_{B}^{\pi}}$

with corrections of order $\left(x_{B}^{\pi} m\right)^{2} / Q^{2}$. In our numerical calculations we use the exact expression of $t_{\pi 0}$, so that any $\phi_{\pi}$ dependent term exactly vanishes at the kinematical point $t_{\pi}=t_{\pi 0}$, where $\phi_{\pi}$ is not defined. We see from (25) that to avoid resonances contributions in the $\pi n$ channel it is advantageous to have low $x_{\pi}$ and relatively large $\left|t_{\pi}\right|$ (while still respecting the condition $\left|t_{\pi}\right| \ll Q^{2}$ for Bjorken kinematics).

A different process relevant in our context is deep inelastic scattering with a leading neutron in the target hemisphere, $e p \rightarrow e n+X$. In the one-pion exchange approximation, this gives access to inclusive deep inelastic scattering $\gamma^{*} \pi \rightarrow X$ and thus provides information of the parton densities of the pion. There is a number of theoretical investigations focusing on very high energies, as achieved in the HERA collider experiments [60-62]. In particular, the studies [63-65] have investigated the role of $\rho$ and also of $a_{2}$ exchange in the framework of Regge theory. Furthermore, rescattering of the neutron has been studied in [65-68] and is typically found to be non-negligible even for $Q^{2}$ of several $\mathrm{GeV}^{2}$. Given the high-energy limit underlying these investigations, we find it difficult to assess the situation for deeply virtual Compton scattering at significantly lower energies.

In summary, we find that existing theoretical investigations of similar processes cannot readily be used to quantify effects beyond the one-pion exchange approximation for $e p \rightarrow e \gamma \pi n$. They emphasize, however, the importance of taking $|t|$ as small as possible. Working at low $x_{\pi}$ will in addition help to avoid resonance effects between the outgoing neutron and pion. The incorporation of $\rho$ exchange into the theoretical analysis should be practically feasible (at least at the level of estimates) if one could gain some information on the size of the $\rho \rightarrow \pi$ transition GPDs, for instance from lattice calculations.

\subsection{Compton scattering on the pion}

Let us now recall the basics of the process $e \pi \rightarrow e \gamma \pi$ in the Bjorken limit, which have been elaborated in detail in earlier work [49]. The analysis of this reaction proceeds in close analogy to the well-known case of a proton target [40, 41]. It is in fact simpler because the pion has spin zero and thus involves fewer GPDs and form factors than the proton. Throughout this section, we retain only the leading terms in the $1 / Q$ expansion, unless explicitly indicated.

We decompose the amplitude for $e \pi \rightarrow e \gamma \pi$ into contributions from Compton scattering and from the Bethe-Heitler process,

$\mathcal{M}_{\text {e } \pi}=\mathcal{M}_{\mathrm{VCS}}+\mathcal{M}_{\mathrm{BH}}$

The corresponding decomposition of the differential cross section for $e \pi \rightarrow e \gamma \pi$ and thus also for $e p \rightarrow e \gamma \pi n$ reads

$d \sigma=d \sigma_{\mathrm{VCS}}+d \sigma_{\mathrm{BH}}+d \sigma_{\mathrm{INT}}$

where $d \sigma_{\text {INT }}$ is the interference term between the BetheHeitler and Compton processes. In the Bjorken limit we have for the squared Compton amplitude

$\sum_{\text {spin }}\left|\mathcal{M}_{\mathrm{VCS}}\right|^{2}=\frac{4 e^{6}}{Q^{2}} \frac{1-y_{\pi}+y_{\pi}^{2} / 2}{y_{\pi}^{2}}\left|\mathcal{H}_{\pi}\right|^{2}$,

with

$$
\begin{aligned}
\mathcal{H}_{\pi}\left(\xi, t_{\pi}\right)= & \sum_{q} e_{q}^{2} \int_{-1}^{1} d x H_{\pi}^{q}\left(x, \xi, t_{\pi}\right) \\
& \times\left[\frac{1}{\xi-x-i \varepsilon}-\frac{1}{\xi+x-i \varepsilon}\right]
\end{aligned}
$$

at leading order in $\alpha_{s}$. Here $H_{\pi}^{q}$ is the GPD for quark flavor $q$ in a $\pi^{+}$as defined in [7], $e_{q}$ is the quark charge in units of the positron charge $e$, and $\sum_{\text {spin }}$ sums over the polarization of the final-state photon. The skewness variable is given by

$\xi=\frac{x_{B}^{\pi}}{2-x_{B}^{\pi}}$.

The squared Bethe-Heitler amplitude can be written as

$$
\begin{aligned}
\sum_{\text {spin }}\left|\mathcal{M}_{\mathrm{BH}}\right|^{2}= & \frac{16 e^{6}}{P} \frac{1-x_{B}^{\pi}}{\left(x_{B}^{\pi}\right)^{2}} \frac{t_{\pi 0}-t_{\pi}}{t_{\pi}^{2}} \\
& \times \frac{1-y_{\pi}+y_{\pi}^{2} / 2}{1-y_{\pi}}\left[F_{\pi}\left(t_{\pi}\right)\right]^{2},
\end{aligned}
$$

where the factor 


$$
\begin{aligned}
P & =\frac{-s^{\prime} u^{\prime}}{Q^{4}\left(1-y_{\pi}\right) / y_{\pi}^{2}} \\
& =\frac{\left(A_{s}-B \cos \phi_{\pi}\right)\left(A_{u}-B \cos \phi_{\pi}\right)}{Q^{4}\left(1-y_{\pi}\right) / y_{\pi}^{2}}
\end{aligned}
$$

with $s^{\prime}=\left(l^{\prime}+q^{\prime}\right)^{2}$ and $u^{\prime}=\left(l-q^{\prime}\right)^{2}$ comes from the lepton propagators. This factor is unity in the Bjorken limit, but it can deviate quite significantly in experimentally relevant kinematics. Up to relative corrections of order $x_{B}^{\pi} m^{2} / Q^{2}$, one has

$A_{s}=\frac{Q^{2}-\left(1-y_{\pi}\right) t_{\pi}}{y_{\pi}}$,

$A_{u}=\frac{\left(1-y_{\pi}\right) Q^{2}-t_{\pi}}{y_{\pi}}$,

$B=\frac{2 Q}{y_{\pi}} \sqrt{\left(1-y_{\pi}\right)\left(1-x_{B}^{\pi}\right)\left(t_{\pi 0}-t_{\pi}\right)}$,

so that for $4\left(1-x_{B}^{\pi}\right)\left(t_{\pi 0}-t_{\pi}\right) \sim\left(1-y_{\pi}\right) Q^{2}$ one can have $P$ close to zero. In our numerical calculations we use the exact expressions of $s^{\prime}$ and $u^{\prime}$ in (33). The interference term between Compton scattering and the Bethe-Heitler process reads

$$
\begin{aligned}
& \sum_{\text {spin }} \mathcal{M}_{\mathrm{VCS}}^{*} \mathcal{M}_{\mathrm{BH}}+\text { c.c. } \\
& =e_{\ell} \frac{16 e^{6}}{P} \frac{\sqrt{1-x_{B}^{\pi}}}{x_{B}^{\pi}} \frac{\sqrt{t_{\pi 0}-t_{\pi}}}{Q t_{\pi}} \\
& \quad \times F_{\pi}\left(t_{\pi}\right)\left[\frac{1-y_{\pi}+y_{\pi}^{2} / 2}{y_{\pi} \sqrt{1-y_{\pi}}} \cos \phi_{\pi} \operatorname{Re} \mathcal{H}_{\pi}\right. \\
& \left.+P_{\ell} \frac{1-y_{\pi} / 2}{\sqrt{1-y_{\pi}}} \sin \phi_{\pi} \operatorname{Im} \mathcal{H}_{\pi}\right],
\end{aligned}
$$

where $e_{\ell}= \pm 1$ is the charge of the lepton beam and $P_{\ell}=$ \pm 1 its helicity.

For lack of better knowledge, we will ignore the offshellness of the incoming pion when evaluating $F_{\pi}\left(t_{\pi}\right)$ and $H_{\pi}^{q}\left(x, \xi, t_{\pi}\right)$. In kinematical factors we take, however, the virtuality $t$ of the initial pion instead of $m_{\pi}^{2}$. As can be seen in (26), this has an important effect on $t_{\pi 0}$ and thus on the factors $t_{\pi 0}-t_{\pi}$ in (32) and (35). The approximation (22), where the $t$-dependence of $d \sigma(e \pi \rightarrow e \gamma \pi)$ is neglected, should therefore be used with caution, especially for small $\left|t_{\pi}\right|$.

In the Bjorken limit, the Bethe-Heitler process dominates over Compton scattering (unless $y_{\pi}$ is very small). This is because $\left|\mathcal{M}_{\mathrm{VCS}}\right|^{2} /\left|\mathcal{M}_{\mathrm{BH}}\right|^{2} \sim t_{\pi} / Q^{2}$ according to (29) and (32). In this situation, privileged access to the Compton amplitude is provided by the interference term (35), which can be separated from the cross section by reversing the beam charge $e_{\ell}$ or the beam helicity $P_{\ell}$. We remark that there are also $P_{\ell}$ dependent terms in $d \sigma_{\mathrm{VCS}}$. As can be seen in [49] they are, however, suppressed by $1 / Q$ compared with the dominant term given in (29).

To conclude this section we remark on the process en $\rightarrow$ $e \gamma \pi^{-} p$, which is accessible through incoherent scattering on nuclear targets. Comparing the subprocesses $e \pi^{+} \rightarrow$ $e \gamma \pi^{+}$and $e \pi^{-} \rightarrow e \gamma \pi^{-}$, we find that the amplitudes for the Bethe-Heitler process have opposite sign, whereas those for the Compton process are equal. This is because the $\gamma \pi \pi$ three-point function changes sign under charge conjugation while the $\gamma \pi \pi \gamma$ four-point function remains the same, and therefore holds even beyond the leading approximation in $1 / Q$. As a consequence, the relations

$d \sigma_{\mathrm{VCS}}\left(e n \rightarrow e \gamma \pi^{-} p\right)=d \sigma_{\mathrm{VCS}}\left(e p \rightarrow e \gamma \pi^{+} n\right)$,

$d \sigma_{\mathrm{BH}}\left(e n \rightarrow e \gamma \pi^{-} p\right)=d \sigma_{\mathrm{BH}}\left(e p \rightarrow e \gamma \pi^{+} n\right)$,

$d \sigma_{\mathrm{INT}}\left(e n \rightarrow e \gamma \pi^{-} p\right)=-d \sigma_{\mathrm{INT}}\left(e p \rightarrow e \gamma \pi^{+} n\right)$

hold as long as the one-pion exchange approximation is valid, whereas they will be invalid if for instance interference between $\pi$ and $\rho$ exchange is important. In typical fixed-target kinematics $d \sigma_{\mathrm{BH}}$ is much larger than $d \sigma_{\mathrm{INT}}$, so that the relations (37) can principle be tested experimentally: when going from $e p \rightarrow e \gamma \pi^{+} n$ to $e n \rightarrow e \gamma \pi^{-} p$, the overall cross section should approximately remain the same, whereas the beam spin or beam charge asymmetry should change sign. We note that a corresponding consistency check for the one-pion exchange approximation in $e p \rightarrow e \pi^{+} n$ and $e n \rightarrow e \pi^{-} p$ was performed in the extraction [15] of the pion form factor.

\section{The generalized quark distribution of the pion}

In this section we describe the model for the pion GPDs which we will use in our numerical studies. We generate a dependence on the skewness $\xi$ by the model of Musatov and Radyushkin [69, 70],

$$
\begin{aligned}
H_{\pi}^{q}\left(x, \xi, t_{\pi}\right)= & \int_{-1}^{1} d \beta \int_{-1+|\beta|}^{1-|\beta|} d \alpha \delta(x-\beta-\xi \alpha) \\
& \times h_{b}(\beta, \alpha) H_{\pi}^{q}\left(\beta, 0, t_{\pi}\right)
\end{aligned}
$$

with

$h_{b}(\beta, \alpha)=\frac{\Gamma(2 b+2)}{2^{2 b+1} \Gamma^{2}(b+1)} \frac{\left[(1-|\beta|)^{2}-\alpha^{2}\right]^{b}}{(1-|\beta|)^{2 b+1}}$

For the profile parameter we will take either $b=2$ or $b=1$. The forward limit of the GPDs is given by the parton densities in the pion as $H_{\pi}^{q}(x, 0,0)=q_{\pi}(x)$ for $x>0$ and $H_{\pi}^{q}(x, 0,0)=-\bar{q}_{\pi}(-x)$ for $x<0$. As an input we will take the parameterizations of SMRS [16] or of GRS [17], both at scale $\mu=2 \mathrm{GeV}$. 


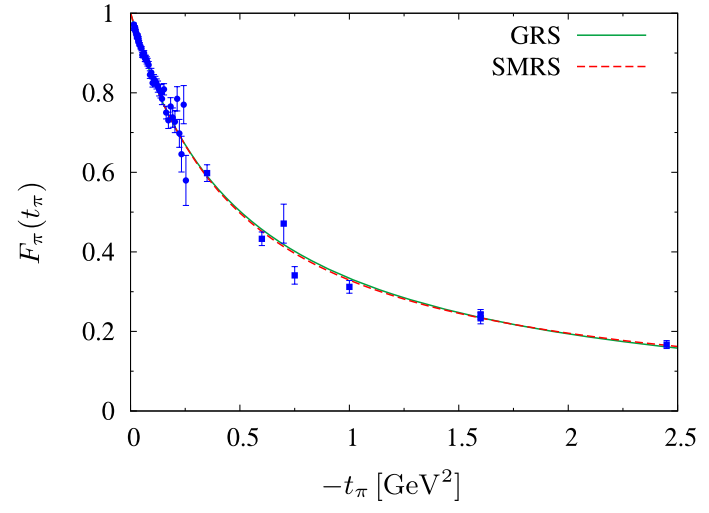

Fig. 3 Data for the electromagnetic pion form factor from [10-15], compared to a fit specified by (41), (42), and the parameters in (43)

The simplest way to model the $t_{\pi}$ dependence is a factorized ansatz

$H_{\pi}^{q}\left(x, 0, t_{\pi}\right)=H_{\pi}^{q}(x, 0,0) F_{\pi}\left(t_{\pi}\right)$,

which automatically satisfies the sum rule

$\sum_{q} e_{q} \int_{-1}^{1} d x H_{\pi}^{q}\left(x, 0, t_{\pi}\right)=F_{\pi}\left(t_{\pi}\right)$.

Both theoretical considerations [71] and lattice QCD calculations $[30,31]$ indicate, however, that the dependence of the GPDs on $t_{\pi}$ and $x$ is correlated. As a model ansatz we will take an exponential $t_{\pi}$ dependence with an $x$ dependent slope. Such an ansatz has proven to be quite successful for the proton [72, 73]. Following [72] we set

$H_{\pi}^{q}\left(x, 0, t_{\pi}\right)=H_{\pi}^{q}(x, 0,0) \exp \left[t_{\pi} f(|x|)\right]$

with

$f(x)=\alpha^{\prime}(1-x)^{3} \ln \frac{1}{x}+B(1-x)^{3}+A x(1-x)^{2}$,

where $\alpha^{\prime}=0.9 \mathrm{GeV}^{-2}$ is taken in accordance with Regge phenomenology. We fit $A$ and $B$ to describe the pion form factor through the sum rule (40), using the data [10-15] as selected in [74]. With the fitted values

$$
\begin{array}{ll}
A=2.19 \mathrm{GeV}^{-2}, & B=-0.38 \mathrm{GeV}^{-2} \quad \text { for GRS } \\
A=1.35 \mathrm{GeV}^{-2}, & B=0.58 \mathrm{GeV}^{-2} \quad \text { for SMRS }
\end{array}
$$

we get a good description for $F_{\pi}\left(t_{\pi}\right)$ with both parameterizations of the parton densities, as shown in Fig. 3 . An intuitive physical interpretation of the function $f(x)$ is obtained in the impact parameter representation [2]: for $x>0$ the average of the squared transverse distance between the quark and the center of momentum of the pion

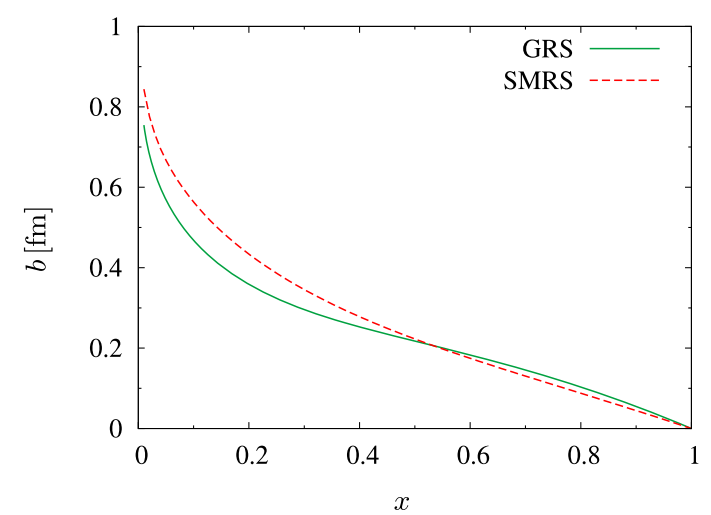

Fig. 4 The average transverse distance $\sqrt{\left\langle b^{2}\right\rangle_{x}}$ between a $u$-quark and the center of momentum of the $\pi^{+}$, as obtained in the two fits of Fig. 3

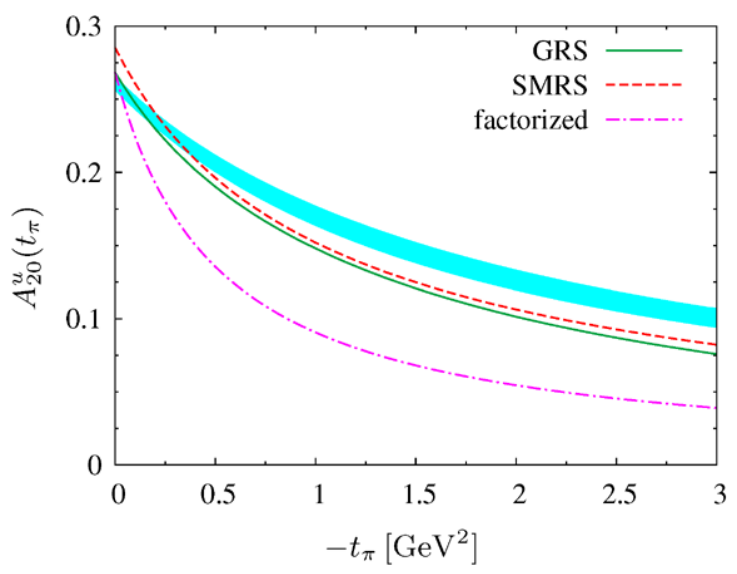

Fig. 5 The second moment (44) of the pion GPD $H_{\pi}^{u}$, obtained with the two fits of Fig. 3. Also shown is the result of the factorized ansatz (39) with the GRS parton densities. The band corresponds to a parameterization of lattice results given in [31], $A_{20}^{u}\left(t_{\pi}\right)=A_{20}^{u}(0) /\left(1-t_{\pi} / M^{2}\right)$ with $A_{20}^{u}(0)=0.261(5)$ and $M=1.37(7) \mathrm{GeV}$

is $\left\langle b^{2}\right\rangle_{x}=4 f(x)$. The results of our two fits are rather similar and yield physically reasonable values, as shown in Fig. 4.

As a caveat we note that the sum rule (40) only constrains the valence quark distributions, given by $H^{q}\left(x, 0, t_{\pi}\right)+$ $H^{q}\left(-x, 0, t_{\pi}\right)=\left[q_{\pi}(x)-\bar{q}_{\pi}(x)\right] \exp \left[t_{\pi} f(x)\right]$ for $x>0$, and is insensitive to the sea quarks. Since sea quarks mix with gluons under evolution, one may expect that the $t_{\pi}$ dependence in this sector is different from the one for valence distributions. The ansatz (41) with a common $t_{\pi}$ dependence for valence and sea quarks (including the strange sea) may hence be regarded as oversimplified. Given, however, that even in the forward limit the sea quark distributions in the pion are poorly known at present, we deem this ansatz to be acceptable for our purpose. 
Using (41) and (42) we can also evaluate the second moment

$A_{20}^{u}\left(t_{\pi}\right)=\int_{-1}^{1} d x x H_{\pi}^{u}\left(x, 0, t_{\pi}\right)$

which has been evaluated in lattice QCD [30, 31]. In Fig. 5 we compare the results of our fits (43) with a monopole parameterization of the lattice data given in [31]. Although not perfect, the agreement is quite good, and certainly much better than the result of the factorized ansatz (39). We note that, in our parameterization, the contribution of sea quarks to the moment (44) is below $30 \%$ at $t_{\pi}=0$ and smaller at nonzero $t_{\pi}$.

Notwithstanding the success of the ansatz given by (41) and (42) in reproducing the lowest two moments of the pion GPD, some cautionary remarks from the theoretical side are in order. As discussed in [72], the asymptotic behavior of the pion form factor at large negative $t$ in our ansatz is controlled by the Feynman mechanism and given by the Drell-Yan relation $F_{\pi}(t) \sim|t|^{-(1+\beta) / 2}$. Here $\beta$ describes the behavior $q(x) \sim(1-x)^{\beta}$ of the parton densities in the limit $x \rightarrow 1$ and is predicted to be $\beta=2$ for the pion [75-77]. Asymptotically the Feynman mechanism hence gives a contribution $F_{\pi}(t) \sim|t|^{-3 / 2}$ to the form factor, which is power suppressed compared with the contribution $F_{\pi}(t) \sim|t|^{-1}$ from the hard-scattering mechanism [75, 76, 78], where it is understood that both power laws are modified by logarithms. The ansatz (41), (42) does not have the form generated by the hard-scattering mechanism for $H_{\pi}^{q}(x, \xi, t)$ at large $t$ [79, 80]. However, it turns out that the parton density parameterizations $[16,17]$ at $\mu=2 \mathrm{GeV}$ both have a large- $x$ power $\beta \approx 1$ within less than $10 \%$, in stark contrast with the prediction $\beta=2$ from power counting. Thus, our ansatz gives $F_{\pi}(t) \sim|t|^{-1}$ at large negative $t$, which is compatible with the monopole behavior that describes very well the available data [74]. We shall not pursue this issue further, and use our ansatz for $H_{\pi}^{q}\left(x, \xi, t_{\pi}\right)$ as a simple candidate form that is not in contradiction with phenomenological constraints.

\section{Cross section estimates}

In this section, we calculate the cross section for $e p \rightarrow$ $e n \gamma \pi$ and its dependence on the beam charge and beam helicity. Since the unpolarized cross section is dominated by the Bethe-Heitler process, the corresponding results are largely model independent (as long as the one-pion exchange approximation is adequate). On the other hand, we will see that the charge and polarization asymmetries for the lepton beam are quite sensitive to the model we assume for the pion GPD. We will compare four simple model scenarios:
1. a skewness dependence generated by (37) and (38) with $b=2$ and a $t_{\pi}$ dependence given by (41) to (43), with the GRS parton densities in the pion,

2. the same as model 1, but with the SMRS parton densities,

3. the same as model 1 , but with $b=1$ instead of $b=2$,

4. the same as model 1 , but with a factorizing $t_{\pi}$ dependence (39). We use this model for the sake of contrast, although it is disfavored by theoretical considerations and lattice data.

For the pion form factor we use a monopole parameterization $F_{\pi}\left(t_{\pi}\right)=1 /\left(1-t_{\pi} / M^{2}\right)$ with $M=714 \mathrm{MeV}$. This provides a very good description of the experimental data, as shown in [74].

As explained in Sect. 2, we impose minimal values for $Q^{2}$ and $s_{\pi}$ and at the same time a maximal value for $|t|$, which requires a sufficiently large energy. We therefore concentrate on typical kinematics for HERMES and for the planned Jefferson Lab upgrade to $11 \mathrm{GeV}$ beam energy. The leading-twist interpretation of DVCS demands that $\left|t_{\pi}\right| \ll$ $Q^{2}$, so that we also put a cut $\left|t_{\pi}\right|<\left|t_{\pi}\right|_{\max }$. We finally impose a maximum value on $y$ (and thus on $y_{\pi}$ ). On the experimental side, this ensures that the scattered lepton has sufficient energy to be detected and identified. On the theoretical side, this improves the $1 / Q$ expansion underlying the approximate formulae (32) and (35), since there are subleading terms in $1 / Q$ that come with a factor $1 / \sqrt{1-y_{\pi}}$ relative to the leading terms. An example of such a subleading term is found in the propagator factor $P$, see (33) and (34).

Let us first consider the case of HERMES, with a beam energy $E_{e}=27.6 \mathrm{GeV}$ in the proton rest frame. We impose a lower limit $\vartheta_{\gamma}>2.57^{\circ}$ on the angle between the momenta of the final-state photon and the lepton beam in the target rest frame. This value corresponds to the maximal geometric acceptance of the electromagnetic calorimeter in the experiment, see e.g. Sect. 5.22 of [81]. Taking limiting values

$Q_{\min }^{2}=2 \mathrm{GeV}^{2}, \quad s_{\pi \min }=4 \mathrm{GeV}^{2}, \quad y_{\max }=0.85$

and

$|t|_{\max }=0.5 \mathrm{GeV}^{2}, \quad\left|t_{\pi}\right|_{\max }=0.9 \mathrm{GeV}^{2}$,

we find a Bethe-Heitler cross section of $\sigma_{\mathrm{BH}}=1620 \mathrm{fb}$, which is between $15 \%$ and $20 \%$ smaller than the result we obtain in model 1 for $\sigma_{\mathrm{BH}}+\sigma_{\mathrm{VCS}}+\sigma_{\mathrm{INT}}$ with either an electron or positron beam. With an integrated luminosity of order $1 \mathrm{fb}^{-1}$ for HERMES running on a proton target [82], we deem this cross section to be too small, since it will be further decreased by experimental acceptance cuts and detection efficiency, and since according to (35) the extraction of the beam charge or beam polarization asymmetry requires a differential measurement at least in the angle $\phi_{\pi}$. Loosening 

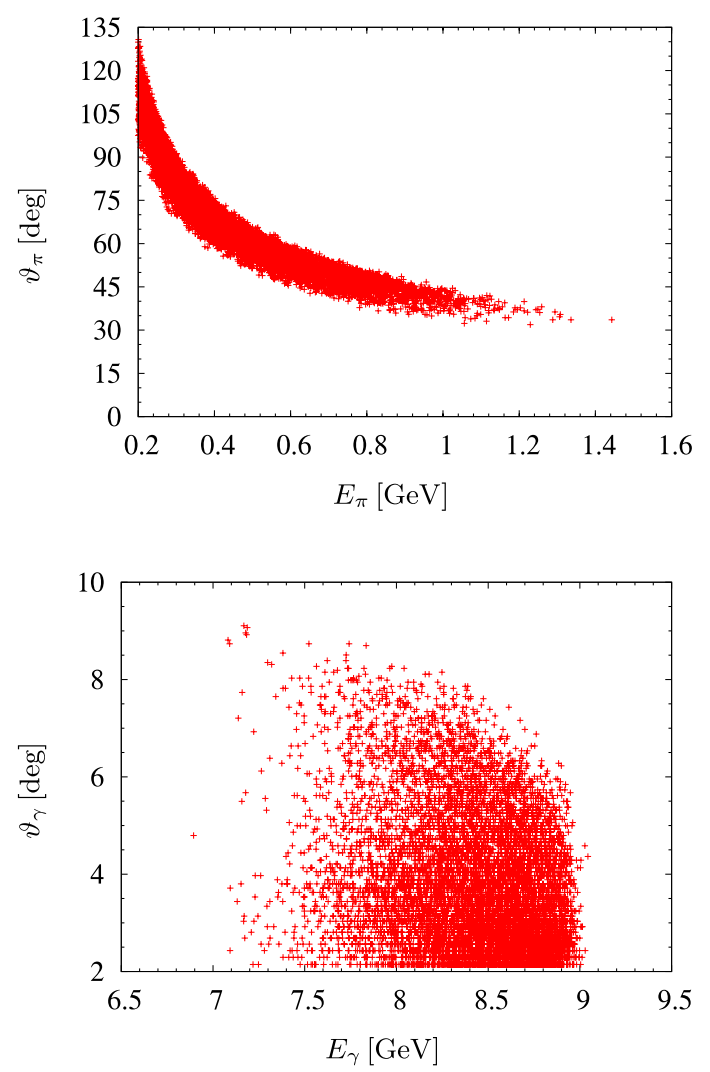

Fig. 6 Scatter plots for the energies and polar angles of the final-state pion and photon in the proton rest frame. The plots are generated from the Bethe-Heitler cross section within the kinematics specified by (45), (49), and (50)

the requirements (45) or (46) would increase the rate at the price of going to kinematics where the theoretical interpretation used in this paper becomes increasingly questionable.

Higher luminosities than at HERMES can be achieved by the experiments at Jefferson Lab. With a currently available beam energy of up to $E_{e}=6 \mathrm{GeV}$, the requirements (45) and (46) leave no available phase space, as can be seen from the bounds on $x_{\pi}$ in (14) and (16). This will be changed with the energy upgrade to $E_{e}=11 \mathrm{GeV}$, which we consider in the remainder of this section. We assume that the outgoing electron, photon, and pion are detected experimentally. Note that identification of the pion (or of the recoiling neutron) is necessary to distinguish the signal process $e p \rightarrow e \gamma \pi^{+} n$ from DVCS on the proton, $e p \rightarrow e \gamma p$, which has a far greater rate. In the proton rest frame we have

$$
\begin{aligned}
E_{\pi}= & \frac{1}{2 m_{N} x_{\pi}}\left\{m_{\pi}^{2}-t_{\pi}-\left[\left(1-x_{\pi}\right)\left(1-x_{B}^{\pi}\right)-x_{B}^{\pi}\right] t\right. \\
+ & 2 \cos \left(\phi_{\pi}+\psi_{e}-\psi_{n}\right) \sqrt{\left(1-x_{\pi}\right)\left(1-x_{B}^{\pi}\right)} \\
& \left.\times \sqrt{\left(t_{0}-t\right)\left(t_{\pi 0}-t_{\pi}\right)}\right\}+\mathcal{O}\left(\frac{m^{2}}{Q}\right),
\end{aligned}
$$
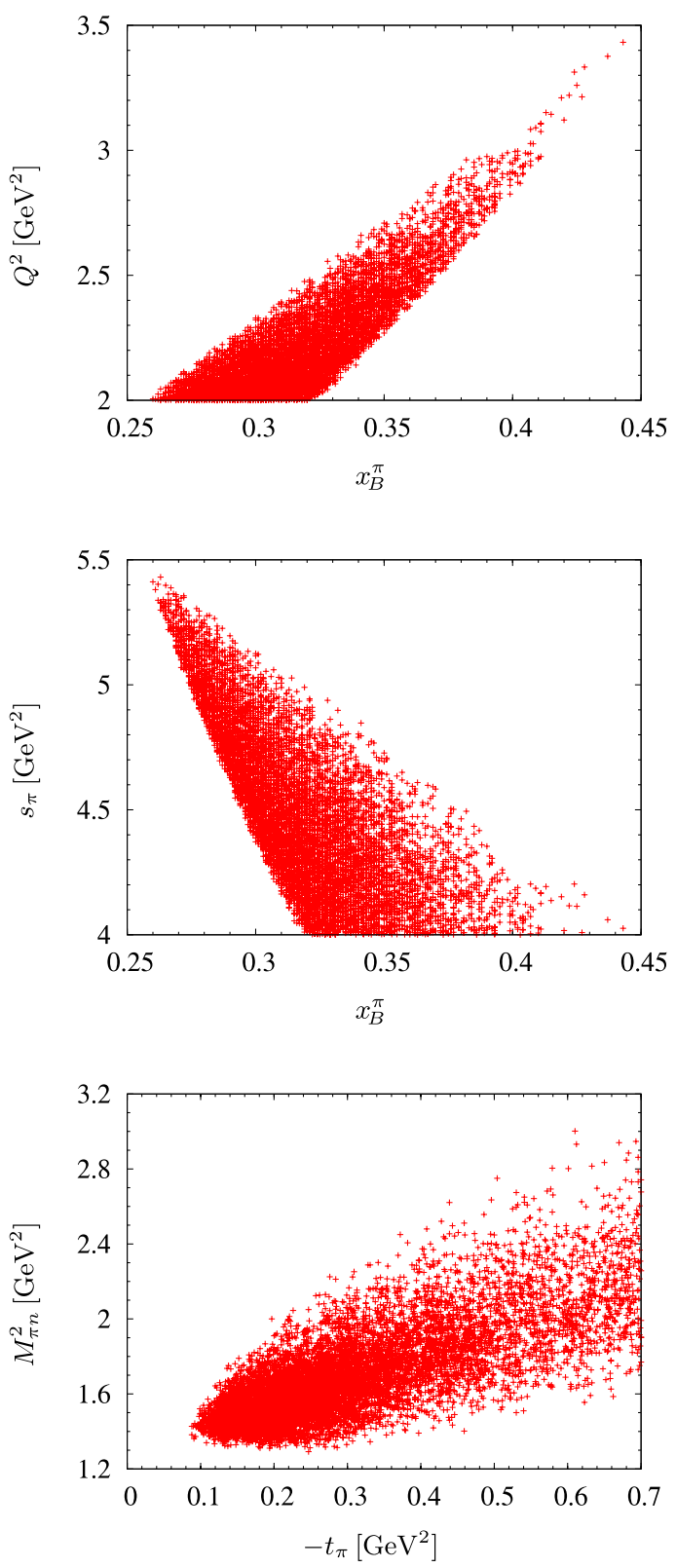

Fig. 7 Scatter plots for various kinematic quantities, generated in the same way as in Fig. 6

$\cos \vartheta_{\pi}=\left(1-\frac{x_{\pi}\left(1-x_{B}^{\pi}\right) m_{N}}{E_{\pi}}\right) \frac{E_{\pi}}{\sqrt{E_{\pi}^{2}-m_{\pi}^{2}}}+\mathcal{O}\left(\frac{m}{Q}\right)$,

where $E_{\pi}$ is the energy of the final-state pion and $\vartheta_{\pi}$ the angle between its momentum and the lepton beam direction. Likewise, we find

$$
\begin{aligned}
& E_{\gamma}=\frac{Q^{2}}{2 x_{B} m_{N}}+\mathcal{O}(m), \\
& \cos \vartheta_{\gamma}=1-\frac{2(1-y) x_{B}^{2} m_{N}^{2}}{Q^{2}}+\mathcal{O}\left(\frac{m^{3}}{Q^{3}}\right)
\end{aligned}
$$


Table 1 The contributions of the Bethe-Heitler and Compton processes and of their interference to the integrated cross section, as well as the weighted cross sections defined in (51). Results are evaluated in model 1 for different kinematic constraints in addition to the cuts (49). The signs of $\sigma_{\text {INT }}$ and $S_{L}^{\sin \phi_{\pi}}$ refer to an electron beam $\left(e_{\ell}=-1\right)$. Limiting values of $Q^{2}, s_{\pi}, t, t_{\pi}$, and $M_{\pi n}^{2}$ are given in units of $\mathrm{GeV}^{2}$, and cross sections in units of $\mathrm{fb}$

\begin{tabular}{|c|c|c|c|c|c|c|c|c|c|c|}
\hline$Q_{\min }^{2}$ & $s_{\pi \min }$ & $|t|_{\max }$ & $\left|t_{\pi}\right|_{\max }$ & $y_{\max }$ & $M_{\pi n \min }^{2}$ & $\sigma_{\mathrm{BH}}$ & $\sigma_{\mathrm{VCS}}$ & $\sigma_{\text {INT }}$ & $S_{C}^{\cos \phi_{\pi}}$ & $S_{L}^{\sin \phi_{\pi}}$ \\
\hline 2 & 4 & 0.3 & 0.7 & 0.85 & - & 18.4 & 0.88 & -0.18 & 0.39 & 7.57 \\
\hline 2 & 4 & 0.3 & 0.7 & 0.8 & - & 5.12 & 0.29 & -0.09 & 0.17 & 2.17 \\
\hline 2 & 4 & 0.3 & 0.7 & 0.9 & - & 45.6 & 1.86 & -0.27 & 0.64 & 17.9 \\
\hline 2 & 4 & 0.2 & 0.7 & 0.85 & - & 0.41 & 0.016 & -0.002 & 0.004 & 0.16 \\
\hline 2 & 4 & 0.5 & 0.7 & 0.85 & - & 105 & 6.52 & -2.32 & 5.00 & 46.2 \\
\hline 2.5 & 4 & 0.3 & 0.7 & 0.85 & - & 2.55 & 0.103 & -0.010 & 0.018 & 0.96 \\
\hline 2 & 5 & 0.3 & 0.7 & 0.85 & - & 0.30 & 0.013 & -0.003 & 0.008 & 0.12 \\
\hline 2 & 4 & 0.3 & 0.5 & 0.85 & - & 16.2 & 0.69 & -0.09 & 0.18 & 6.30 \\
\hline 2 & 4 & 0.3 & 0.7 & 0.85 & 1.5 & 13.4 & 0.67 & -0.19 & 0.42 & 5.72 \\
\hline 2 & 4 & 0.3 & 0.7 & 0.85 & 1.8 & 5.08 & 0.31 & -0.14 & 0.30 & 2.46 \\
\hline
\end{tabular}

for the energy of the outgoing photon and its polar angle. To estimate the cross section and its dependence on the beam charge and polarization, we assume some minimal experimental cuts, which correspond to the acceptance planned for the CLAS++ detector [83],

$E_{e}^{\prime}>500 \mathrm{MeV}, \quad 8^{\circ}<\vartheta_{e}<45^{\circ}$,

$E_{\gamma}>100 \mathrm{MeV}, \quad 2^{\circ}<\vartheta_{\gamma}<40^{\circ}$,

$E_{\pi}>200 \mathrm{MeV}, \quad 5^{\circ}<\vartheta_{\pi}<135^{\circ}$.

Typical values of these quantities are shown in the scatter plots of Fig. 6. These plots have been generated using the Bethe-Heitler cross section, which dominates the total rate as we shall see shortly. We have imposed the kinematic requirements (45) and

$|t|_{\max }=0.3 \mathrm{GeV}^{2}, \quad\left|t_{\pi}\right|_{\max }=0.7 \mathrm{GeV}^{2}$,

where compared with (46) we have taken smaller $|t|_{\max }$ and $\left|t_{\pi}\right|_{\max }$, so that the one-pion exchange and the leading-twist approximations are better fulfilled. We see that the pion has small to intermediate energy and covers a large angular region, whereas the photon is energetic and strongly focused in the beam direction. In the corresponding scatter plot for the outgoing electron kinematics (not shown here) we have $1.6 \mathrm{GeV}<E_{e}^{\prime}<3.4 \mathrm{GeV}$ and $13^{\circ}<\vartheta_{e}<25^{\circ}$, so that the experimental cuts on these quantities in (49) have no influence.

Figure 7 shows corresponding scatter plots for other relevant variable pairs. In the first two panels we see the values of $Q^{2}, s_{\pi}$, and $x_{B}^{\pi}$ at which the Compton process $\gamma^{*} \pi \rightarrow$ $\gamma \pi$ can be probed with $E_{e}=11 \mathrm{GeV}$. The last panel shows that typical values of the squared $\pi n$ invariant mass $M_{\pi n}^{2}$ are between $1.3 \mathrm{GeV}^{2}$ and $2.2 \mathrm{GeV}^{2}$, which unfortunately includes the region of nucleon resonances. We will come back to this point shortly.
In addition to the contributions $\sigma_{\mathrm{BH}}, \sigma_{\mathrm{VCS}}, \sigma_{\mathrm{INT}}$ of the different subprocesses and their interference to the integrated cross section, we evaluate the weighted differences

$$
\begin{aligned}
& S_{C}^{\cos \phi_{\pi}}=\int d \phi_{\pi} \cos \phi_{\pi}\left[\frac{d \sigma\left(e_{\ell}=+1\right)}{d \phi_{\pi}}-\frac{d \sigma\left(e_{\ell}=-1\right)}{d \phi_{\pi}}\right], \\
& S_{L}^{\sin \phi_{\pi}}=\int d \phi_{\pi} \sin \phi_{\pi}\left[\frac{d \sigma\left(P_{\ell}=+1\right)}{d \phi_{\pi}}-\frac{d \sigma\left(P_{\ell}=-1\right)}{d \phi_{\pi}}\right]
\end{aligned}
$$

of cross sections for different beam charge or beam polarization. In the approximation given by (29), (32), (35), they are respectively proportional to $\operatorname{Re} \mathcal{H}_{\pi}$ and $\operatorname{Im} \mathcal{H}_{\pi}$ in the interference term. In the Bjorken limit, the propagator factor $P$ in (33) becomes $\phi_{\pi}$ independent, so that $S_{C}^{\cos \phi_{\pi}}$ and $S_{L}^{\sin \phi_{\pi}}$ respectively give the coefficients of $\cos \phi_{\pi}$ and $\sin \phi_{\pi}$ in $2 \pi d \sigma_{\mathrm{INT}} / d \phi_{\pi}$. With $E_{e}=11 \mathrm{GeV}$ and the kinematics delineated by (45) and (50), we find, however, a clear $\phi_{\pi}$ dependence for $P$. For this reason, the interference term (35) also contributes to the cross section integrated over $\phi_{\pi}$, as seen in Table 1 . We also recall that beyond the leading approximation in $1 / Q$, the weighted cross section $S_{L}^{\sin \phi_{\pi}}$ receives a contribution from $d \sigma_{\mathrm{VCS}}$ in addition to the one from $d \sigma_{\text {INT. }}$. This can be seen from the expressions in [49] and is well-known in the case of DVCS on a proton [40, 41].

In Table 1 we show our results for the integrated and weighted cross sections calculated in model 1 for different kinematic constraints. For the choice in (45) and (50), shown in the first row, one obtains an integrated cross section of $18.4 \mathrm{fb}$. With an estimated luminosity of $3000 \mathrm{fb}^{-1}$ per year at $\mathrm{CLAS}++$, this gives a very comfortable rate of about 55000 events, so that one may hope that even with realistic experimental cuts and detection efficiencies there will be sufficient statistics to perform a binning in several variables. 
The further entries in Table 1 illustrate how the situation changes if one modifies our baseline kinematic constraints. Raising $y_{\max }$ from 0.85 to 0.9 would more than double the rate, but as discussed above this would make the $1 / Q$ expansion underlying the formulae (32) and (35) worse. Lowering instead $y_{\max }$ from 0.85 to 0.8 , would reduce the rate by a factor of about 3.5 . Increasing $|t|_{\max }$ from $0.3 \mathrm{GeV}^{2}$ to $0.5 \mathrm{GeV}^{2}$ we obtain a significantly higher rate, but the onepion exchange approximation is much more problematic in that case. Decreasing $|t|_{\max }$ to $0.2 \mathrm{GeV}^{2}$ would improve the quality of the one-pion exchange approximation, but the resulting loss of rate is probably too much for such a cut to be useful. Taking a stricter cut of $2.5 \mathrm{GeV}^{2}$ instead of $2 \mathrm{GeV}^{2}$ for $Q_{\min }^{2}$ would make the leading-twist analysis of DVCS safer but decrease the cross section by about a factor of 7 . An even stronger decrease is found if one requires $s_{\pi}$ to be above $5 \mathrm{GeV}^{2}$ instead of $4 \mathrm{GeV}^{2}$. By contrast, only very little rate is lost if one takes $0.5 \mathrm{GeV}^{2}$ instead of $0.7 \mathrm{GeV}^{2}$ for $\left|t_{\pi}\right|_{\text {max }}$, so that it may be worthwhile to consider a stronger cut on this variable. Finally, one may wish to impose a cut on the invariant mass of the $\pi n$ system, so as to reduce possible resonance effects in this channel, which are not taken into account in our theoretical description. The entries in the last two rows of Table 1 respectively correspond to a lower cut on $M_{\pi n}$ at the mass of the $\Delta$ and at the mass of the $\Delta$ plus its width. The resulting rates show that at least part of the kinematic region where resonance effects may spoil a simple interpretation can be removed in an analysis without losing too much signal.

In Fig. 8 we show the Bethe-Heitler cross section differential in $t, t_{\pi}$, or in $x_{\pi}$. We see that in the kinematics of Jefferson Lab at $11 \mathrm{GeV}$ a cutoff $|t|_{\text {max }}$ much below $0.3 \mathrm{GeV}^{2}$ severely restricts the available phase space. The $t_{\pi}$ spectrum falls off much more slowly than the corresponding $t$ spectrum for DVCS on the proton, which readily follows from the slower decrease of the pion electromagnetic form factor compared with the proton form factors. Nevertheless, the falloff for $\left|t_{\pi}\right| \gtrsim 0.2 \mathrm{GeV}^{2}$ is sufficiently steep to account for the weak dependence of the cross section on $\left|t_{\pi}\right|_{\max }$ seen in Table 1 . The spectrum in $x_{\pi}$ is rather featureless and reflects the form of the pion flux factor in Fig. 2 together with the phase space boundaries $x_{\min }$ and $x_{\max }$ given in (14) and (16).

The sensitivity to the pion GPDs of the Compton cross section and the interference term is documented in Table 2 for the different models introduced above. To obtain a more detailed picture, we plot in Fig. 9 the weighted cross sections (51) differential in $t, t_{\pi}$, and $x_{\pi}$. We see that the spread between models is much more pronounced for $S_{C}^{\cos \phi_{\pi}}$, with different signs and even a zero crossing in $t_{\pi}$ for some of the models. By contrast, the variation of $S_{L}^{\sin \phi_{\pi}}$ is less drastic and concerns the size of the weighted cross section more than its dependence on $t, t_{\pi}$, or $x_{\pi}$. On the other hand, the beam spin asymmetry $S_{L}^{\sin \phi_{\pi}}$ is significantly larger than the
Table 2 As Table 1 but for different models of the pion GPDs. The kinematic constraints in (45), (49), and (50) are always assumed. The corresponding Bethe-Heitler cross sections is $\sigma_{\mathrm{BH}}=18.4 \mathrm{fb}$

\begin{tabular}{llrrr}
\hline Model & $\sigma_{\text {VCS }}$ & \multicolumn{1}{c}{$\sigma_{\text {INT }}$} & $S_{C}^{\cos \phi_{\pi}}$ & $S_{L}^{\sin \phi_{\pi}}$ \\
\hline 1 & 0.88 & -0.18 & 0.39 & 7.57 \\
2 & 0.67 & -0.66 & 1.57 & 6.44 \\
3 & 1.12 & -0.21 & 0.43 & 8.57 \\
4 & 0.70 & 0.25 & -0.62 & 6.78 \\
\hline
\end{tabular}
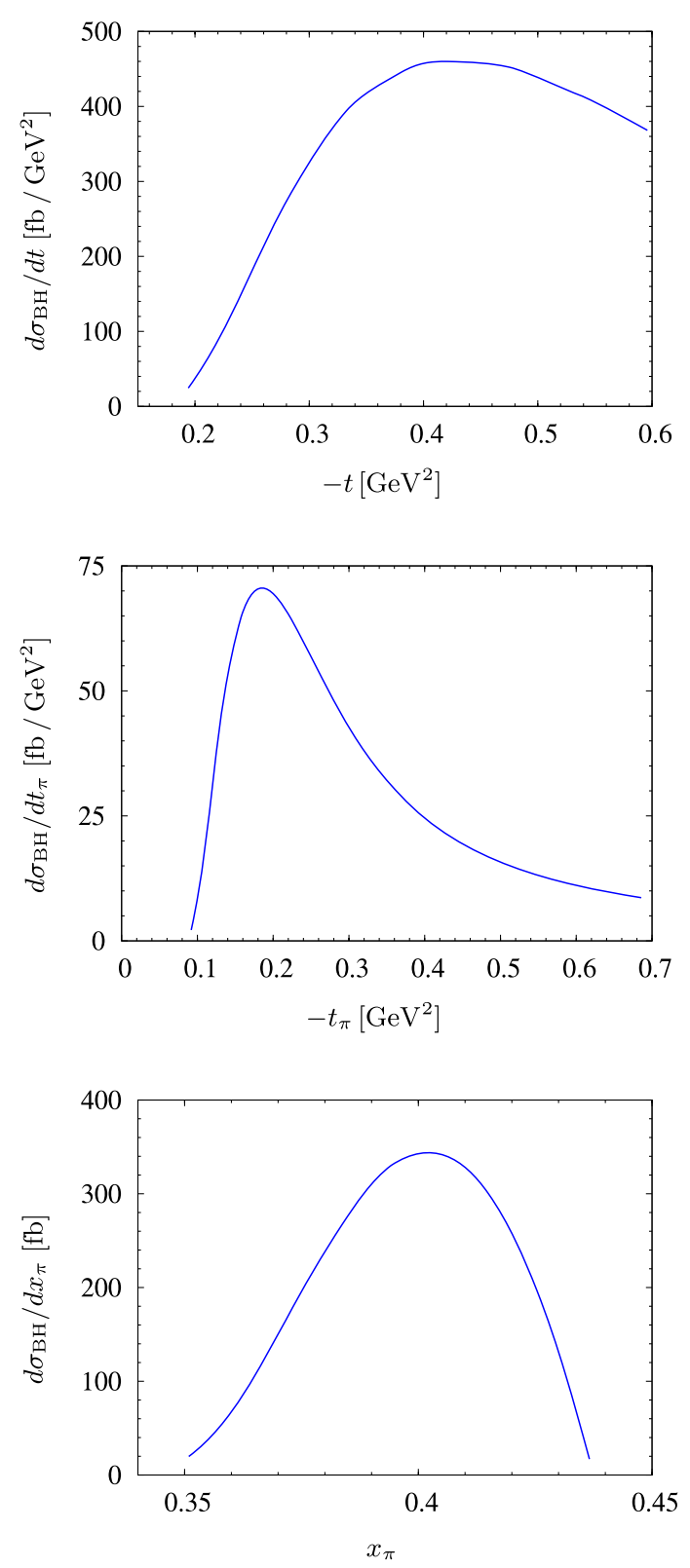

Fig. 8 The Bethe-Heitler cross section taken differential in $t, t_{\pi}$, or $x_{\pi}$, and calculated with the cuts specified by (45), (49), and (50). For the cross section differential in $t$ we have omitted the first constraint in (50) 

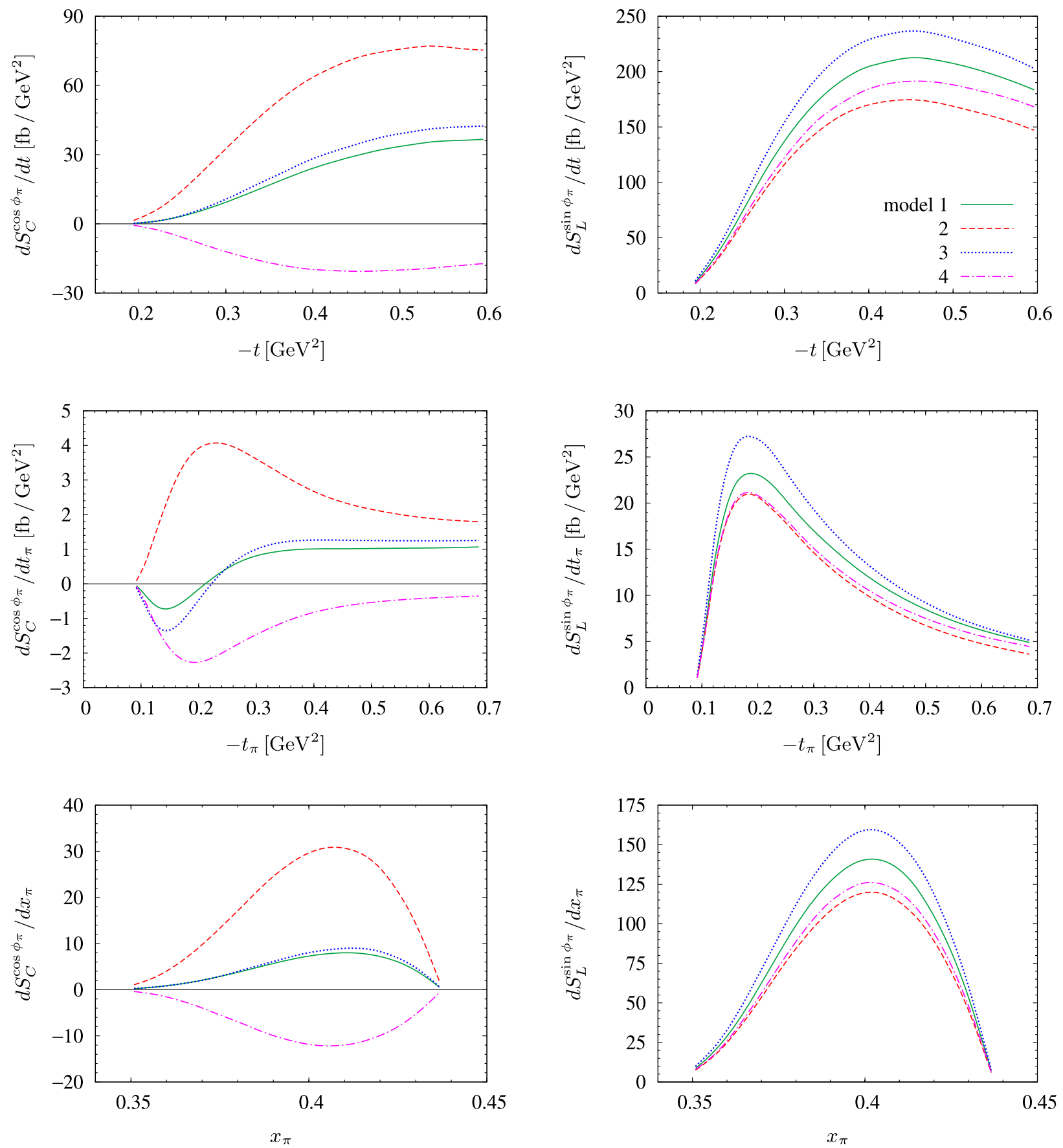

Fig. 9 As Fig. 8 but for the weighted cross sections (51), calculated with the four GPD models specified in the text. The sign of $S_{L}^{\sin \phi_{\pi}}$ refers to an electron beam

beam charge asymmetry $S_{C}^{\cos \phi_{\pi}}$ and may be easier to measure in practice.

\section{Summary}

We have investigated the possibility to study DVCS on the pion in the reaction $e p \rightarrow e \gamma \pi^{+} n$. Such a study is exper- imentally demanding for several reasons. Firstly, the phase space is limited by the requirements of small $t$ on one side (so that pion exchange dominates the process and the pion is not too far off-shell) and of large $Q^{2}$ and $s_{\pi}$ on the other side (so that an analysis based on the Bjorken limit is applicable). As can be seen from (14) and (16), this favors experiments with a higher ep c.m. energy. Secondly, the cross section for $e p \rightarrow e \gamma \pi^{+} n$ is significantly smaller than the one for 
$e p \rightarrow e \gamma p$, which puts high demands on both the luminosity and the experimental identification of the final state.

We find that conditions for an experimental study of this process are not favorable in current experiments, with HERMES being limited by the available event rate and Jefferson Lab by the beam energy. After the planned energy upgrade to $11 \mathrm{GeV}$ at Jefferson Lab, it should, however, be possible to investigate the reaction in detail. Using acceptance cuts relevant for the CLAS++ detector, we find comfortable event rates for kinematical conditions that may not be optimal but should be adequate for a first look at DVCS on the pion in the Bjorken regime and at intermediate values of the skewness $\xi$. Using simple models for the GPDs of the pion, we estimate that information about them could be provided both by the beam spin and by the beam charge asymmetry, with the latter showing a more pronounced sensitivity to the ansatz for the GPDs but being smaller in size. Optimized studies might be feasible one day at a projected electron-ion collider [84, 85], where in particular it should be possible to take the invariant mass of the $\pi n$ system above the resonance region.

As a part of our model study, we have explored an ansatz for the pion GPDs that depends exponentially on $t_{\pi}$ with a slope decreasing with $x$. Taking a functional form previously used for the nucleon [72] together with current parameterizations of the parton densities in the pion, we obtain an excellent fit to the experimental data of the electromagnetic pion form factor. Without adjusting further parameters, the ansatz then compares rather well with recent results from lattice QCD for the second Mellin moment of the GPDs.

Acknowledgements We gratefully thank H. Avagyan, A. Bacchetta, J. Bartels, D. Brömmel, M. Guidal, D. Hasch, M. Kopytin, C. Riedl, W. Kugler, S. Niccolai, B. Pire, J. Stewart and L. Szymanowski for useful discussions and correspondence. Special thanks go to B. Pire for useful remarks on the manuscript. This work is supported by the exchange program PROCOPE of the German Academic Exchange Service and the French Ministère des Affaires Étrangères, by the Integrated Infrastructure Initiative of the European Union (contract number RII3-CT-2004-506078), and by the Helmholtz Association (contract number VH-NG-004). D.A. and J.P.L. thank the members of the IFPA group at the University of Liège for their hospitality.

\section{References}

1. X.D. Ji, J. Phys. G 24, 1181 (1998). hep-ph/9807358

2. M. Burkardt, Int. J. Mod. Phys. A 18, 173 (2003). hep-ph/0207047

3. J.P. Ralston, B. Pire, Phys. Rev. D 66, 111501 (2002). hep-ph/0110075

4. M. Diehl, Eur. Phys. J. C 25, 223 (2002) [Erratum: Eur. Phys. J. 31, 277 (2003)]. hep-ph/0205208

5. A.V. Belitsky, X.D. Ji, F. Yuan, Phys. Rev. D 69, 074014 (2004). hep-ph/0307383

6. K. Goeke, M.V. Polyakov, M. Vanderhaeghen, Prog. Part. Nucl. Phys. 47, 401 (2001). hep-ph/0106012

7. M. Diehl, Phys. Rep. 388, 41 (2003). hep-ph/0307382
8. A.V. Belitsky, A.V. Radyushkin, Phys. Rep. 418, 1 (2005). hep-ph/0504030

9. S. Boffi, B. Pasquini, Riv. Nuovo Cimento. 30, 387 (2007). arXiv:0711.2625

10. S.R. Amendolia et al. (NA7 Collaboration), Nucl. Phys. B 277, 168 (1986)

11. H. Ackermann et al., Nucl. Phys. B 137, 294 (1978)

12. P. Brauel et al., Z. Phys. C 3, 101 (1979)

13. J. Volmer et al. (Jefferson Lab $F_{\pi}$ Collaboration), Phys. Rev. Lett. 86, 1713 (2001). nucl-ex/0010009

14. V. Tadevosyan et al. (Jefferson Lab $F_{\pi}$ Collaboration), Phys. Rev. C 75, 055205 (2007). nucl-ex/0607007

15. T. Horn et al. (Jefferson Lab $F_{\pi}-2$ Collaboration), Phys. Rev. Lett. 97, 192001 (2006). nucl-ex/0607005

16. P.J. Sutton, A.D. Martin, R.G. Roberts, W.J. Stirling, Phys. Rev. D 45, 2349 (1992)

17. M. Glück, E. Reya, I. Schienbein, Eur. Phys. J. C 10, 313 (1999). hep-ph/9903288

18. K. Wijesooriya, P.E. Reimer, R.J. Holt, Phys. Rev. C 72, 065203 (2005). nucl-ex/0509012

19. I.V. Anikin, A.E. Dorokhov, A.E. Maksimov, L. Tomio, V. Vento, Nucl. Phys. A 678, 175 (2000). hep-ph/9905332

20. A.P. Bakulev, R. Ruskov, K. Goeke, N.G. Stefanis, Phys. Rev. D 62, 054018 (2000). hep-ph/0004111

21. H.M. Choi, C.R. Ji, L.S. Kisslinger, Phys. Rev. D 64, 093006 (2001). hep-ph/0104117

22. H.M. Choi, C.R. Ji, L.S. Kisslinger, Phys. Rev. D 66, 053011 (2002). hep-ph/0204321

23. A. Mukherjee, I.V. Musatov, H.C. Pauli, A.V. Radyushkin, Phys. Rev. D 67, 073014 (2003). hep-ph/0205315

24. B.C. Tiburzi, G.A. Miller, Phys. Rev. D 67, 013010 (2003). hep-ph/0209178

25. B.C. Tiburzi, G.A. Miller, Phys. Rev. D 67, 113004 (2003). hep-ph/0212238

26. L. Theussl, S. Noguera, V. Vento, Eur. Phys. J. A 20, 483 (2004). nucl-th/0211036

27. W. Broniowski, E. Ruiz Arriola, Phys. Lett. B 574, 57 (2003). hep-ph/0307198

28. F. Bissey, J.R. Cudell, J. Cugnon, J.P. Lansberg, P. Stassart, Phys. Lett. B 587, 189 (2004). hep-ph/0310184

29. W. Broniowski, E.R. Arriola, K. Golec-Biernat, Phys. Rev. D 77, 034023 (2008). arXiv:0712.1012

30. D. Brömmel et al. (QCDSF/UKQCD Collaboration), PoS LAT2005, 360 (2006). hep-lat/0509133

31. D. Brömmel, PhD thesis, Universität Regensburg, 2007, DESYTHESIS-2007-023

32. D. Brömmel et al. (QCDSF Collaboration), arXiv:0708.2249

33. S. Dalley, Phys. Lett. B 570, 191 (2003). hep-ph/0306121

34. M.V. Polyakov, C. Weiss, Phys. Rev. D 60, 114017 (1999). hep-ph/9902451

35. O.V. Teryaev, Phys. Lett. B 510, 125 (2001). hep-ph/0102303

36. I.V. Anikin, B. Pire, O.V. Teryaev, Phys. Rev. D 62, 071501 (2000). hep-ph/0003203

37. A.V. Belitsky, D. Müller, Nucl. Phys. B 589, 611 (2000). hep-ph/0007031

38. N. Kivel, M.V. Polyakov, A. Schäfer, O.V. Teryaev, Phys. Lett. B 497, 73 (2001). hep-ph/0007315

39. A.V. Radyushkin, C. Weiss, Phys. Rev. D 63, 114012 (2001). hep-ph/0010296

40. M. Diehl, T. Gousset, B. Pire, J.P. Ralston, Phys. Lett. B 411, 193 (1997). hep-ph/9706344

41. A.V. Belitsky, D. Müller, A. Kirchner, Nucl. Phys. B 629, 323 (2002). hep-ph/0112108

42. A. Airapetian et al. (HERMES Collaboration), Phys. Rev. Lett. 87, 182001 (2001). hep-ex/0106068.

43. S. Stepanyan et al. (CLAS Collaboration), Phys. Rev. Lett. 87, 182002 (2001). hep-ex/0107043 
44. A. Airapetian et al. (HERMES Collaboration), Phys. Rev. D 75, 011103 (2007). hep-ex/0605108

45. C. Muñoz Camacho et al. (Jefferson Lab Hall A Collaboration), Phys. Rev. Lett. 97, 262002 (2006). nucl-ex/0607029

46. F.X. Girod et al. (CLAS Collaboration), Phys. Rev. Lett. 100, 162002 (2008). arXiv:0711.4805

47. A. Airapetian et al. (HERMES Collaboration), J. High Energy Phys. 0806, 066 (2008). arXiv:0802.2499

48. H1 Collaboration, Abstract 179 submitted to EPS 2007, Manchester, July 2007, H1 prelim-07-011. http://www-h1.desy.de/ h1/www/publications/conf/list.EPS2007.html

49. A.V. Belitsky, D. Müller, A. Kirchner, A. Schäfer, Phys. Rev. D 64, 116002 (2001). hep-ph/0011314

50. B. Pire, L. Szymanowski, Phys. Rev. D 71, 111501 (2005). hep-ph/0411387

51. J.P. Lansberg, B. Pire, L. Szymanowski, Phys. Rev. D 73, 074014 (2006). hep-ph/0602195

52. J.P. Lansberg, B. Pire, L. Szymanowski, arXiv:0709.2567

53. E.R. Berger, M. Diehl, B. Pire, Eur. Phys. J. C 23, 675 (2002). hep-ph/0110062

54. A. Bacchetta, U. D'Alesio, M. Diehl, C.A. Miller, Phys. Rev. D 70, 117504 (2004). hep-ph/0410050

55. V.G.J. Stoks, R. Timmermans, J.J. de Swart, Phys. Rev. C 47, 512 (1993). nucl-th/9211007

56. W. Koepf, L.L. Frankfurt, M. Strikman, Phys. Rev. D 53, 2586 (1996). hep-ph/9507218

57. M. Guidal, J.M. Laget, M. Vanderhaeghen, Nucl. Phys. A 627, 645 (1997)

58. M. Diehl, Eur. Phys. J. C 19, 485 (2001). hep-ph/0101335

59. J.P. Lansberg, B. Pire, L. Szymanowski, Phys. Rev. D 75, 074004 (2007) [Erratum: Phys. Rev. D 77, 019902 (2008)]. hep-ph/0701125

60. C. Adloff et al. (H1 Collaboration), Eur. Phys. J. C 6, 587 (1999). hep-ex/9811013

61. S. Chekanov et al. (ZEUS Collaboration), Nucl. Phys. B 637, 3 (2002). hep-ex/0205076

62. S. Chekanov et al. (ZEUS Collaboration), Nucl. Phys. B 776, 1 (2007). hep-ex/0702028.

63. B. Kopeliovich, B. Povh, I. Potashnikova, Z. Phys. C 73, 125 (1996). hep-ph/9601291
64. H. Holtmann, G. Levman, N.N. Nikolaev, A. Szczurek, J. Speth, Phys. Lett. B 338, 393 (1995). hep-ph/9602229

65. V.A. Khoze, A.D. Martin, M.G. Ryskin, Eur. Phys. J. C 48, 797 (2006). hep-ph/0606213

66. N.N. Nikolaev, J. Speth, B.G. Zakharov, hep-ph/9708290

67. U. D’Alesio, H.J. Pirner, Eur. Phys. J. A 7, 109 (2000). hep-ph/9806321

68. A.B. Kaidalov, V.A. Khoze, A.D. Martin, M.G. Ryskin, Eur. Phys. J. C 47, 385 (2006). hep-ph/0602215

69. A.V. Radyushkin, Phys. Rev. D 59, 014030 (1999). hep-ph/ 9805342

70. I.V. Musatov, A.V. Radyushkin, Phys. Rev. D 61, 074027 (2000). hep-ph/9905376

71. M. Burkardt, Phys. Lett. B 595, 245 (2004). hep-ph/0401159

72. M. Diehl, T. Feldmann, R. Jakob, P. Kroll, Eur. Phys. J. C 39, 1 (2005). hep-ph/0408173

73. M. Guidal, M.V. Polyakov, A.V. Radyushkin, M. Vanderhaeghen, Phys. Rev. D 72, 054013 (2005). hep-ph/0410251

74. D. Brömmel et al. (QCDSF/UKQCD Collaboration), Eur. Phys. J. C 51, 335 (2007). hep-lat/0608021

75. G.P. Lepage, S.J. Brodsky, Phys. Rev. D 22, 2157 (1980)

76. S.J. Brodsky, G.P. Lepage, in Perturbative Quantum Chromodynamics, ed. by A.H. Mueller. Adv. Ser. Direct. High Energy Phys., vol. 5 (World Scientific, Singapore, 1989), p. 93

77. F. Yuan, Phys. Rev. D 69, 051501 (2004). hep-ph/0311288

78. A.V. Efremov, A.V. Radyushkin, Phys. Lett. B 94, 245 (1980)

79. C. Vogt, Phys. Rev. D 64, 057501 (2001) [Erratum: Phys. Rev. D 69 (2004) 079901]. hep-ph/0101059

80. P. Hoodbhoy, X.D. Ji, F. Yuan, Phys. Rev. Lett. 92, 012003 (2004). hep-ph/0309085

81. Z. Ye, Ph.D. thesis, Universität Hamburg, 2007, DESY-THESIS2007-005

82. J. Stewart, private communication, 2007

83. M. Guidal, S. Niccolai, private communication, 2007

84. A. Deshpande, R. Milner, R. Venugopalan, W. Vogelsang, Ann. Rev. Nucl. Part. Sci. 55, 165 (2005). hep-ph/0506148

85. C. Aidala et al. (The EIC Working Group), A High Luminosity, High Energy Electron-Ion-Collider, White Paper, April 2007. http://web.mit.edu/eicc/Documentation.html 